\title{
Programas para agresores de violencia de género en prisión: ¿De qué evidencia disponemos?
}

\section{Rehabilitation programs for perpetrators of gender violence in prison: What kind of evidences do we have?}

Recibido el 10 noviembre 2015/Publicado el 5 abril 2016

\author{
Bárbara Sordi ${ }^{1}$ \\ Universidad de Sevilla
}

\section{RESUMEN}

El objetivo de esta revisión es sintetizar el conocimiento científico sobre los programas de rehabilitación para agresores de violencia de género desarrollados en los centros penitenciarios de España. Para ello, se ha realizado una búsqueda exhaustiva de los trabajos publicados entre 2001-2015 y, tras aplicar los criterios de inclusión/exclusión, se han seleccionado un total de 9 estudios. Los resultados obtenidos pueden definirse como muy positivos en las evaluaciones postratamiento, si bien son escasos los estudios sobre reincidencia y que sean objeto de seguimiento a largo plazo. Las conclusiones indican que, pese a no existir datos concluyentes sobre los resultados de los programas, hay que continuar apostando por las intervenciones específicas intramuros.

Palabras clave: Programas de rehabilitación, violencia de género, prisión.

\footnotetext{
1 La correspondencia debe enviarse a Bárbara Sordi Stock. Instituto Andaluz Interuniversitario de Criminología, Universidad de Sevilla. Avda. Enramadilla, 18-20, 41018. Sevilla. España.: barbarasordistock@gmail.com
} 


\begin{abstract}
The aim of this review is to summarize the scientific knowledge about rehabilitation programs of gender violence developed in Spanish prisons. In order to achieve this goal, I have completed an exhaustive research including a great quantity of scientific papers written in the period 2001-2015, and after to apply the inclusion/exclusion standards, we have selected a total number of 9 articles. Positive results have been achieved in post-treatment evaluations. On the other hand, we have found just a few studies that deal with recidivism and also a few that include long term follow-up. Our conclusions suggest that despite the lack of conclusive data about the results of the programs it seems advisable to keep on supporting the intervention of specific gender violence in prison.
\end{abstract}

Keywords: Rehabilitation programs, gender violence, prison.

\title{
1. Introducción
}

Según los datos oficiales de la Secretaría General de Instituciones Penitenciarias (en adelante SGIP), en julio de 2015 la población de reclusos por delitos y faltas relacionados con la violencia de género en las cárceles de España arroja un total de 4.183 hombres (SGIP, 2015). Tras los cambios legales introducidos por la Ley Orgánica 1/2004, dicha violencia ha pasado a ser la tercera forma más frecuente de ingreso en prisión $^{2}$ (SGIP, 2010a) correspondiendo a la Administración Penitenciara enfrentar un nuevo reto: realizar programas intramuros para reclusos por la comisión de crímenes relacionados con la violencia de género. Si bien la posibilidad de asumir el tratamiento de los internos como una tarea prioritaria podría generar considerables interrogantes respecto a la capacidad de los establecimientos penitenciarios, el resultado no ha sido otro que la oficialización de estos programas por la Secretaría General de Instituciones Penitenciarias y por el Departamento de Justicia de Cataluña.

Los programas bajo competencia de la SGIP se han estructurado fundamentalmente en tres etapas: $1^{\circ}$ ) 2001 - 2002: programa piloto; $2^{\circ}$ ) 2004 - 2010: Programa de Tratamiento en Prisión para agresores en el Ámbito Familiar; $3^{\circ}$ ) 2010actualidad: Violencia de Género: Programa de Intervención para Agresores - PRIA. La justificación para la definitiva creación de un programa específico intramuros -el Programa de Tratamiento en Prisión para agresores en el Ámbito Familiar (SGIP,

${ }^{2}$ Los delitos contra la salud pública y contra el patrimonio y el orden socioeconómico (hurtos, robos estafas etc.) encabezan los índices de encarcelamiento (Véase SGIP, 2015). 
2005)- ha encontrado respaldo no solo en el aumento considerable de internos por delitos de esta naturaleza, sino en las características que estas personas presentan cuando se les compara con la población carcelaria en general, entre las que destacan: riesgo de reincidencia moderadamente alto como consecuencia de cogniciones muy consolidadas; ausencia de carrera criminal (2 de cada 3 eran primarios); diagnóstico de psicopatía significativo; deseabilidad social mayor de la esperada para las personas que están en la cárcel (Fernández-Montalvo \& Echeburúa, 2005). El citado programa se ha revelado más adecuado que la experiencia piloto (2001/2002), en atención a su capacidad de enfocar las necesidades concretas de los agresores de violencia de género (Fernández-Montalvo \& Echeburúa, 2005). Asimismo, se ha dado especial énfasis al hecho de que los agresores tienden a negar, minimizar o trasladar la culpa de los hechos y de la perpetuación del conflicto a sus parejas (Echeburúa, Fernández-Montalvo \& Amor, 2006). Sin embargo, no todas las cárceles han incorporado el programa tal cual se ha diseñado, sirviendo más bien como un referente para las intervenciones que comenzaban a ejecutarse intramuros ${ }^{3}$.

Hoy en día, las cárceles bajo competencia de la SGIP cuentan con Programa Marco, cuyo diseño se recoge en un Manual titulado Violencia de Género: Programa de Intervención para Agresores más conocido como PRIA (SGIP, 2010). El propósito de este programa es perseguir "la extinción de cualquier tipo de conducta violenta dirigida hacia la pareja, así como la modificación de todo tipo de actitudes y creencias de tipo sexista” (SGIP, 2010, p. 7). Se trata de una intervención grupal, con 50 sesiones que se desarrollan a lo largo de un año. Los encuentros son semanales con una duración aproximada de dos horas y media. El programa contempla evaluación pre y postratamiento, así como seguimiento de los resultados. Como novedad en relación a los programas anteriormente desarrollados, el PRIA propone una intervención cognitivo-conductual desde la perspectiva de género. Se trata de una innovación en la orientación que ha introducido cuestiones diferenciales como: 1) La combinación de aspectos clínicos con la perspectiva de género; 2) Énfasis en la motivación inicial de los agresores; 3) Examen de las diferentes conductas que integran la violencia de género

\footnotetext{
3 Sirva de ejemplo el convenio entre el Gobierno de Navarra e Instituciones Penitenciarias para desarrollar un programa con reclusos dentro de la prisión de Pamplona (Echauri, Romero \& Rodríguez, 2005) y la experiencia del Centro Penitenciario de Monterroso-Lugo en Galicia (Gutiérrez, 2009).
} 
(por ejemplo, la violencia psicológica y la instrumentalización de los hijos). La SGIP, un año más tarde de la edición del Manual, publica un anexo titulado El Delito de Violencia de Género y los Penados Extranjeros (SGIP, 2011), cuyo propósito es afrontar la realidad multicultural existente en el ámbito penitenciario.

En el caso de Cataluña, el año 2009 sería un punto de inflexión, en razón del diseño de programas con distintitas intensidades ejecutados en el Centro Penitenciario Brians-2 (Loinaz, 2009). Habitualmente, la metodología aplicada es grupal y se realizan sesiones individuales de apoyo o seguimiento. También contempla evaluación pre y postratamiento y la valoración clínica del progreso del sujeto en la terapia. El programa considerado "corto" se desarrolla aproximadamente a lo largo de 24 sesiones, siendo dos sesiones semanales de dos horas, mientras que el programa considerado "largo" se desarrolla por lo general a lo largo de 48 sesiones (Leganés, 2012). Aunque se trate de programas dirigidos a distintas tipologías de agresores, ambos se desenvuelven en base a bloques comunes y abordan temas como el ciclo de la violencia, las distorsiones cognitivas y el control emocional, la baja autoestima, los celos, el estilo de vida saludable, la asertividad y comunicación y la resolución de problemas.

Al contrario de lo que se podría pensar, la puesta en marcha de un Programa Marco o de programas de distintas intensidades no ha imposibilitado la creación de otros programas con diseños diversificados (duración, enfoque, etc.). Cabe citar como ejemplo el programa con enfoque emocional (PREMOVIGE) en una cárcel de Jaén (Rodríguez-Espartal \& Lopez-Zafra, 2013) y la experiencia piloto con condenados por violencia de género que han recibido Terapia de Aceptación y Compromiso (ACT) en una cárcel de Castilla y León (Flores \& Pascual, 2013).

La historia reciente de los programas intramuros para agresores de violencia de género se caracteriza inequívocamente por la pluralidad. Cerca de 3.000 hombres ya han participado de una intervención específica en una cuarentena de centros penitenciarios por todo el país bajo competencia de la SGIP (Véase SGIP, 2012). Además, se han impartido una cantidad nada desdeñable de cursos de formación dirigido a los equipos multidisciplinares que actúan en prisiones, con especial atención a los sociólogos, psicólogos, juristas, pedagogos, trabajadores sociales, educadores y funcionarios de vigilancia (Filardo, 2013; SGIP, 2012). 
A día de hoy, resulta posible reunir algunos estudios sobre las características personales de los sujetos y sobre los resultados de las intervenciones. De una parte, el factor tiempo es un condicionante para la propia existencia de datos, una vez que las valoraciones precisan ser realizadas a largo plazo y la experiencia española es un tanto reciente (Sordi, 2014). De otra parte, los recursos disponibles para las investigaciones sobre programas en prisiones no han crecido en la misma proporción que la población reclusa por esta tipología delictiva (Loinaz, 2011).

Este escenario sugiere la necesidad de ampliar los estudios nacionales para identificar cuáles son las estrategias más adecuadas a las especificidades de la población reclusa. El objetivo de esta revisión, pues, es sintetizar el conocimiento científico sobre los programas para agresores de violencia de género ejecutados en las cárceles de España.

\section{Método}

\subsection{Unidad de análisis}

La unidad de análisis de este estudio son las publicaciones sobre programas de rehabilitación para hombres que se encuentran en situación de prisión por delitos relacionados con violencia de género.

\subsection{Materiales}

Artículos de revistas especializadas, libros, capítulos de libros y tesis de doctorado, todos ellos publicados en papel o por vía electrónica y que se encuentran en los repositorios de universidades, base datos de gran amplitud, entre otros. Concretamente, se han seguido las siguientes estrategias de búsqueda: a) Metabuscadores (i.e. Google Académico); b) Recursos/buscadores de amplitud más restringida a España (i.e. Dialnet, TESEO y Catálogo de la Biblioteca de la Universidad de Sevilla); c) Observatorios de violencia de género y Centros de Investigación (http://www.inmujer.gob.es, www.workwith-perpetrators.eu, www.cienciaspenales.net y http://www.cejfe.cat/index.php); d) Base de datos de Instituciones Penitenciarias (http://www.institucionpenitenciaria.es/ y 
http://justicia.gencat.cat/ca) y otras de alcance más amplio (i.e.PsycInfo, Scopus y Web of Science); e) Eventual contacto con investigadores/as de referencia en la temática (autores/as de artículos u otros/as que también investiguen programas de rehabilitación en España).

\subsection{Diseño y procedimiento}

Se han buscado materiales publicados entre enero de 2001 y julio de 2015. El año de partida coincide con las noticias de la puesta en marcha del programa piloto intramuros para maltratadores y el período de finalización ha tenido por intensión examinar los datos más recientes.

Teniendo en cuenta el objetivo propuesto, las palabras clave de mayor uso en lengua española han sido: programas de reeducación de maltratadores y violencia de género, programas de rehabilitación para maltratadores y violencia de género, programa en prisión, reincidencia y violencia de género, evaluación de programas, tratamiento penitenciario y efectividad. Con el propósito de abarcar las publicaciones en revistas internacionales se han utilizado las siguientes palabras clave en inglés: rehabilitation program gender violence, prison program domestic violence, gender violence and prison. La búsqueda de material se ha llevado a cabo mediante un enfoque multidisciplinario, que va más allá de las fuentes que se centran en la Criminología, pues el tema es objeto de estudio en otros campos científicos, con énfasis en la Psicología, Psiquiatría, Salud Pública, Derecho y Sociología. Además, las referencias bibliográficas de los materiales obtenidos también han sido examinadas con el fin de localizar otras publicaciones pertinentes al tema.

Para evitar la superposición o duplicado de resultados, dado que algunos de los materiales se han encontrado en más de una base de datos/recurso/buscador o han aparecido repetidamente en una misma base de datos/recurso/buscador al utilizar palabras clave diferentes, se ha llevado a cabo un proceso de selección con tal objeto. Los registros generados a través de este procedimiento se han revisado y se han eliminado los que no se ajustaban estrictamente al objetivo del estudio propuesto, así como los que se han referido a la misma muestra de estudio y que han sido publicados 
en distintos medios académicos bajo títulos diversos. De esta forma, a efecto de resultados tan solo se ha considerado un material.

El eventual contacto con investigadores/as de referencia se ha producido de forma ocasional, dando prioridad aquellos/as con mayor número de publicaciones sobre la materia. El mismo fue informal, vía telefónica, email o Skype, resultando de gran utilidad a la hora de evitar la superposición o duplicado de resultados.

Se han considerado como criterios de selección tan solo aquellos estudios sobre programas de rehabilitación para hombres que se encuentran en situación de prisión por delitos relacionados con violencia de género. Se han excluido las investigaciones: a) Que han comparado agresores que habían recibido un programa como pena/medida alternativa con aquellos que habían recibido un programa en medio cerrado; b) Sobre agresores que habían recibido un programa como pena/medida alternativa; c) Sobre agresores sexuales que cumplían pena de prisión; d) Presentadas en forma de ponencias o comunicaciones a congresos así como los editoriales; e) Relacionadas con la Justicia de Menores; y f) Que solo han investigado las características de los reclusos, sin especificar la participación en un programa intramuros. Por tanto, se han incluido tanto aquellos estudios en los cuales la totalidad de la muestra participaba o había participado de un programa, como aquellos en los cuales parte de la muestra participaba o había participado en una intervención.

Los materiales seleccionados han sido exportados a una Tabla Word en la cual se han descrito los datos considerados importantes, como por ejemplo, muestra estudiada, local a que refiere la investigación, objetivos y principales resultados alcanzados, con énfasis en los hallazgos atribuidos al programa.

\section{Resultados}

Se han encontrado un total de 105 publicaciones y, con la aplicación de los criterios recabados al banco de estudios, se han seleccionado un total 9 artículos publicados entre enero de 2001 y julio de 2015, que aparecen reflejados en la tabla 1. 
Tabla 1. Estudios sobre programas de rehabilitación para hombres que se encuentran en situación de prisión por delitos relacionados con violencia de

género

\begin{tabular}{|c|c|c|c|c|c|}
\hline Estudio & Diseño & Muestra & $\begin{array}{l}\text { Participación } \\
\text { en programa }\end{array}$ & Ámbito & $\begin{array}{c}\text { Principales hallazgos y, en particular, cambios atribuidos } \\
\text { al programa }\end{array}$ \\
\hline $\begin{array}{c}\text { Fernández } \\
\text {-Montalvo } \\
\& \\
\text { Echeburúa } \\
\text { (2005) }\end{array}$ & $\begin{array}{c}\text { Estudio descriptivo } \\
\text { de las características } \\
\text { demográficas, penales } \\
\text { y psicopatológicas de } \\
\text { los agresores y } \\
\text { comparativo de las } \\
\text { variables entre } \\
\text { homicidas y no } \\
\text { homicidas. }\end{array}$ & 162 & $\begin{array}{c}\text { Todos } \\
\text { participaron de } \\
\text { un programa. }\end{array}$ & $\begin{array}{c}18 \\
\text { prisiones }\end{array}$ & $\begin{array}{l}\checkmark \text { Subraya la necesidad de diseñar programas específicos } \\
\text { intramuros para violencia de género. } \\
\checkmark \text { Riesgo de reincidencia moderadamente alto en casi la } \\
\text { mitad de la muestra. } \\
\checkmark \text { Tipología delictiva directamente relacionada con el estado } \\
\text { civil de los reclusos: alto porcentaje de divorciados y } \\
\text { solteros. } \\
\checkmark \text { Reclusos con mayor incidencia de antecedentes } \\
\text { psicopatológicos ( } 43,82 \%) \text {, particularmente relacionados con } \\
\text { conductas adictivas e impulsividad, al ser comparados con la } \\
\text { población en general. } \\
\checkmark \text { El } 17,9 \% \text { de la muestra cometió homicidio. No se } \\
\text { establecieron diferencias significativas entre homicidas y no } \\
\text { homicidas. }\end{array}$ \\
\hline
\end{tabular}




\begin{tabular}{|c|c|c|c|c|c|}
\hline $\begin{array}{l}\text { Martínez, } \\
\text { Pérez \& } \\
\text { López } \\
\text { (2008) }\end{array}$ & $\begin{array}{l}\text { Estudio comparativo } \\
\text { entre dos grupos de } \\
\text { sujetos: grupo tratado } \\
\text { y grupo control. } \\
\text { Evalúa la eficacia del } \\
\text { tratamiento } \\
\text { psicológico. }\end{array}$ & 32 & $\begin{array}{l}\text { Todos } \\
\text { participaron de } \\
\text { un programa. } \\
\text { Grupo control } \\
\text { formado por } 30 \\
\text { reclusos. }\end{array}$ & $\begin{array}{c}\text { C.P. Quatre } \\
\text { Camins - } \\
\text { Cataluña }\end{array}$ & $\begin{array}{l}\checkmark \text { Las variables sociodemográficas no presentan diferencias } \\
\text { significativas entre el grupo tratado y el grupo control. } \\
\checkmark \text { Eficacia parcial del programa: diferencias entre el grupo } \\
\text { tratado y el grupo control que podrían vincularse a los } \\
\text { efectos del programa son relevantes en relación a las } \\
\text { distorsiones cognitivas (inferioridad de la mujer y uso de la } \\
\text { violencia como estrategia válida para resolver conflictos), } \\
\text { control de la impulsividad e ira. No hay diferencias } \\
\text { significativas en empatía o estadios de cambio terapéutico. }\end{array}$ \\
\hline $\begin{array}{l}\text { Echeburúa } \\
\quad \& \\
\text { Fernández } \\
\text {-Montalvo } \\
\text { (2009) }\end{array}$ & $\begin{array}{l}\text { Evaluación pre y } \\
\text { postratamiento: } \\
\text { evalúa la efectividad } \\
\text { del tratamiento } \\
\text { psicológico. }\end{array}$ & 148 & $\begin{array}{c}\text { Todos } \\
\text { participaron de } \\
\text { un programa. }\end{array}$ & $\begin{array}{c}18 \\
\text { prisiones }\end{array}$ & $\begin{array}{l}\checkmark \text { Aquellos que finalizaran el tratamiento }(68,2 \%) \\
\text { presentaran: modificación significativa de los sesgos } \\
\text { cognitivos sobre la inferioridad de la mujer; de la violencia } \\
\text { como una estrategia válida en el enfrentamiento de } \\
\text { problemas; mejora en la capacidad de controlar la ira; } \\
\text { reducción de la impulsividad y aumento de la autoestima. } \\
\text { Además, el programa es efectivo para la disminución de la } \\
\text { sintomatología psicopatológica. }\end{array}$ \\
\hline
\end{tabular}




\begin{tabular}{|c|c|c|c|c|c|}
\hline $\begin{array}{c}\text { Martínez } \\
\text { \& Pérez } \\
\text { (2009) }\end{array}$ & $\begin{array}{c}\text { Evaluación pre y } \\
\text { postratamiento: } \\
\text { evalúa la efectividad } \\
\text { del tratamiento } \\
\text { psicológico. }\end{array}$ & 28 & $\begin{array}{c}\text { Todos } \\
\text { participaron de } \\
\text { un programa: } \\
\text { dos grupos de } \\
\text { reclusos (de } 8 \text { y } \\
13 \text { hombres) } \\
\text { participaron de } \\
\text { un programa de } \\
4 \text { meses; los } \\
\text { demás, } \\
\text { participaron de } \\
\text { un programa de } \\
9 \text { meses. }\end{array}$ & $\begin{array}{c}\text { C.P. Quatre } \\
\text { Camins - } \\
\text { Cataluña }\end{array}$ & $\begin{array}{l}\checkmark \text { Aporta evidencias empíricas del efecto del programa en } \\
\text { ciertas variables psicológicas: el programa logra reducir la } \\
\text { impulsividad y, de forma significativa, la expresión de la ira, } \\
\text { así como aumenta el control de los sujetos. A su vez no tiene } \\
\text { un efecto significativo en las distorsiones cognitivas, } \\
\text { habilidades interpersonales y empatía y estadios de cambio } \\
\text { terapéutico. } \\
\checkmark \text { Subrayan la heterogeneidad de los sujetos a los que va } \\
\text { dirigido el programa. Ente otras cuestiones, sostienen la } \\
\text { posibilidad de realizar programas con distintas intensidades } \\
\text { y la necesidad de desarrollar programas para la población } \\
\text { extrajera. }\end{array}$ \\
\hline $\begin{array}{l}\text { SGIP } \\
(2010 a)\end{array}$ & $\begin{array}{c}\text { Estudio descriptivo } \\
\text { de las características } \\
\text { demográficas, } \\
\text { penales, } \\
\text { penitenciarias, } \\
\text { psicopatológicas de } \\
\text { los agresores y su } \\
\text { relación con la } \\
\text { víctima. }\end{array}$ & 387 & $\begin{array}{c}\text { El 26,7\% estaba } \\
\text { participando o } \\
\text { había } \\
\text { participado de } \\
\text { un programa. }\end{array}$ & $\begin{array}{c}29 \\
\text { prisiones }\end{array}$ & $\begin{array}{l}\checkmark \text { Tipología delictiva: el delito de violencia de género, en la } \\
\text { mayoría de los encuestados, es el delito principal en el } \\
\text { historial del penado (“grupo exclusivo" - } 282 \text { internos). En } \\
\text { un grupo minoritario, dicho delito forma parte de un } \\
\text { conjunto de delitos ("grupo antisocial” - } 89 \text { internos). } \\
\checkmark \text { Sugieren relación entre el abandono del programa y el } \\
\text { perfil delictivo del recluso: del total de reclusos que } \\
\text { abandonaron el tratamiento (33\%), solo uno pertenecía al } \\
\text { "grupo exclusivo"; los demás eran todos del "grupo } \\
\text { antisocial”. }\end{array}$ \\
\hline
\end{tabular}




\begin{tabular}{|c|c|c|c|c|c|}
\hline $\begin{array}{l}\text { Loinaz } \\
\text { (2011) }\end{array}$ & $\begin{array}{l}\text { Evaluación pre y } \\
\text { postratamiento, con } \\
\text { seguimiento de parte } \\
\text { de los casos (tiempo } \\
\text { medio de } 15 \text { meses). }\end{array}$ & 50 & $\begin{array}{l}\text { El } 80 \% \text { de la } \\
\text { muestra a la que } \\
\text { se ha realizado } \\
\text { seguimiento ( } 40 \\
\text { reclusos) había } \\
\text { participado de } \\
\text { un programa } \\
\text { antes de su } \\
\text { excarcelación. }\end{array}$ & $\begin{array}{l}\text { C.P. Brians - } \\
2 \text { - Cataluña }\end{array}$ & $\begin{array}{l}\checkmark \text { Reincidencia: } 15 \% \text { (muestra de } 40 \text { hombres). Señala que } \\
\text { no participar de un programa puede suponer una } \\
\text { probabilidad del } 50 \% \text { de reincidir, frente al } 9 \% \text { de los que } \\
\text { recibieron tratamiento. Variables diferenciales } \\
\text { estadísticamente significativas en los reincidentes al ser } \\
\text { comparados con los no reincidentes: trastorno de } \\
\text { personalidad paranoide, estereotipo machista, aceptación de } \\
\text { la violencia, factores de riesgo de conducta antisocial y } \\
\text { hábitos delictivos. } \\
\checkmark \text { Aporta evidencias empíricas sobre la posibilidad de } \\
\text { diseñar intervenciones ajustadas a tipologías de agresores } \\
\text { (muestra de } 50 \text { hombres): Grupo } 1 \text { "violentos solo con la } \\
\text { pareja/estables emocionalmente/integrados socialmente" } \\
\text { (46\%): mayor autoestima, compulsivos, narcisistas, } \\
\text { histriónicos, control elevado de ira. Grupo } 2 \text { "violentos } \\
\text { generalizados/poco estables emocionalmente/no integrados } \\
\text { socialmente" (54\%): maltrato en la infancia, dependencia de } \\
\text { alcohol y drogas, condenas previas, mayor nivel de } \\
\text { distorsiones cognitivas sobre la mujer, impulsivos, falta de } \\
\text { empatía, estilo de apego inseguro y hostil. }\end{array}$ \\
\hline $\begin{array}{l}\text { Rodríguez- } \\
\text { Espartal \& } \\
\text { Lopez- } \\
\text { Zafra } \\
\text { (2013) }\end{array}$ & $\begin{array}{l}\text { Evalúa si el programa } \\
\text { con enfoque } \\
\text { emocional } \\
\text { (PREMOVIGE) } \\
\text { resulta más eficaz que } \\
\text { el cognitivo- } \\
\text { conductual o la } \\
\text { ausencia de }\end{array}$ & 36 & $\begin{array}{l}24 \text { reclusos } \\
\text { participaron de } \\
\text { programas } \\
\text { distintos: } 11 \text { del } \\
\text { cognitivo- } \\
\text { conductual y } 13 \\
\text { del emocional } \\
\text { (PREMOVIGE). }\end{array}$ & $\begin{array}{l}\text { Institución } \\
\text { Penitenciaria } \\
\text { Provincial } \\
\text { de Jaén - } \\
\text { Jaén II }\end{array}$ & $\begin{array}{l}\checkmark \text { La ausencia de tratamiento, sea el PREMOVIGE, sea el } \\
\text { cognitivo-conductual, es perjudicial y supone un riesgo } \\
\text { importante para la reincidencia. } \\
\checkmark \text { Variables diferenciales significativas: mayor descenso de } \\
\text { los pensamientos distorsionados sobre la mujer y sobre el } \\
\text { uso de la violencia, así como mayores expectativas de } \\
\text { cambio en aquellos hombres que participaron del } \\
\text { PREMOVIGE. }\end{array}$ \\
\hline
\end{tabular}




\begin{tabular}{|c|c|c|c|c|c|}
\hline & tratamiento. & & $\begin{array}{c}12 \text { reclusos } \\
\text { asignados al } \\
\text { grupo control. }\end{array}$ & & \\
\hline $\begin{array}{c}\text { Flores \& } \\
\text { Pascual } \\
\text { (2013) }\end{array}$ & $\begin{array}{l}\text { Estudio piloto con } \\
\text { evaluación pre y } \\
\text { postratamiento: } \\
\text { evalúa la aplicación } \\
\text { de la Terapia de } \\
\text { Aceptación y } \\
\text { Compromiso (ACT) } \\
\text { en combinación con } \\
\text { estrategias formales } \\
\text { de mindfulness. }\end{array}$ & 18 & $\begin{array}{c}12 \text { reclusos } \\
\text { participaron del } \\
\text { programa y } 6 \\
\text { fueron } \\
\text { asignados al } \\
\text { grupo control. }\end{array}$ & $\begin{array}{c}\text { C.P. } \\
\text { Comunidad } \\
\text { Autónoma } \\
\text { de Castilla y } \\
\text { León }\end{array}$ & $\begin{array}{l}\checkmark \text { El } 71,4 \% \text { estaba cumpliendo condena solo por violencia } \\
\text { de género. } \\
\checkmark \text { La participación en el programa posibilitó la disminución } \\
\text { de los niveles de evitación e impulsividad así como bajar, } \\
\text { ostensiblemente, el esfuerzo por eliminar el malestar } \\
\text { (aceptación del malestar sin necesidad de reaccionar de una } \\
\text { forma violenta). } \\
\checkmark \text { El } 67 \% \text { completó la totalidad de sesiones del programa. }\end{array}$ \\
\hline
\end{tabular}




\subsection{Características de los agresores en prisión}

Los estudios han presentado conclusiones similares en relación a las características personales de los agresores. Se trata, predominantemente, de hombres españoles con una edad en torno a los 40 años, separados/divorciados, con poca formación educacional y que ocupan puestos de trabajo que exigen poca cualificación.

Sobre la nacionalidad de los reclusos, proporcionalmente, el porcentaje de extranjeros cumpliendo pena de prisión por delitos de violencia de género no dista de forma considerable de la población general extranjera en prisión (Véase García \& DíezRipollés, 2012; SGIP, 2015). Lo interesante de la variable nacionalidad es que puede ser un condicionante para la participación del sujeto en los programas, no por el origen del individuo propiamente, sino porque el idioma y los contenidos a abordar en la intervención exigen otro enfoque (Martínez \& Pérez, 2009; SGIP, 2011).

En relación a la edad, la franja de edad de los penados por violencia de género es ligeramente superior a la edad media de la población reclusa masculina (SGIP, 2010a). Si se compara esta variable con el tiempo de permanencia en prisión, se constata que todas las franjas de edad están contempladas y que el ingreso en prisión de gran parte de los agresores se produce en su juventud - el 55\% de los reclusos han ingresado en prisión con menos de 35 años (SGIP, 2010a).

En lo que concierne al estado civil, se trata en su mayoría de sujetos separados/divorciados. La gran presencia de divorciados/separados que participan de un programa puede deberse al hecho de que sean más conscientes del daño y de las pérdidas como consecuencia del uso de la violencia (SGIP, 2010a). Más de un estudio llama la atención sobre el número de hombres que afirman conservar su status de casado aun estando en prisión (Fernández-Montalvo \& Echeburúa, 2005; SGIP, 2010a). Si por un lado la denuncia de violencia de género puede suponer el inicio de una separación o la separación ser un factor desencadénate de la violencia (Loinaz, 2013), por otro lado no pocas víctimas parecen seguir manteniendo relación de afectividad con el agresor que se encuentra en prisión (Véase Instituto Andaluz de la Mujer, 2012).

Sobre el nivel de estudios, un contraste entre los datos sugiere un aumento en el índice de reclusos con formación que han pasado a integrar el sistema penitenciario 
(Fernández-Montalvo \& Echeburúa, 2005). Sobre el nivel de estudio y su relación con los programas, es importante referir que éste constituye un significativo factor limitador de la aceptación del sujeto en el grupo (Martínez \& Pérez, 2009; SGIP, 2010a).

Por último, sobre la ocupación habitual de los reclusos antes de su entrada en prisión, el hecho de tener un “trabajo poco cualificado” parece redundar en moderados y no en escasos - recursos económicos (Fernández-Montalvo \& Echeburúa, 2005). El análisis conjunto de las investigaciones sugiere que un porcentaje considerable de agresores tiene condiciones económicas bastante razonables.

Las informaciones descritas son útiles porque influyen en el diseño y en los resultados esperados de la intervención rehabilitadora. Ahora bien, se viene estableciendo un consenso de que los datos con mayor trascendencia son los que ofrecen las clasificaciones tipológicas una vez que permiten diseñar programas ajustados a las necesidades reales de los sujetos (Loinaz, Echeburúa \& Torrubia, 2010). En otras palabras, partir de un diseño de intervención que sea adecuado a determinada población tiene incidencia directa en los resultados y, por tanto, en la mayor o menor posibilidad de reincidencia (Loinaz, 2009). Estas son cuestiones claves una vez que muchos de los agresores vuelven con su pareja o iniciarán una nueva relación de pareja (Loinaz, 2009; 2013).

\subsection{Tipologías de agresores}

Los estudios empíricos han demostrado que la violencia de género es una violencia multicausal, fenomenología que implica trabajar con una pluralidad de agresores. Esta realidad no impide que algunos de ellos puedan presentar características similares (Echauri, Fernández-Montalvo, Martínez \& Azcárate, 2011; Fernández-Montalvo, 2009; SGIP, 2010a). Si bien la SGIP se apoya en la tesis de que la violencia de género es un problema cultural que no está asociada al uso de drogas y alcohol y que el agresor es un delincuente ocasional, un porcentaje minoritario - pero no menos importante - de agresores no se incluye en esta hipótesis. La participación de distintos grupos de agresores en un mismo programa ejerce influencia directa en los resultados esperados. La propia SGIP (2010a) ha indicado en este estudio específico que el delito de violencia de género, o bien es el delito principal en el historial del penado ("grupo exclusivo" - 
282 internos), o bien forma parte de un conjunto de delitos (“grupo antisocial” - 89 internos). Estos últimos, por lo general, mantienen un estilo de vida delincuencial en el que la violencia tiene función instrumental y no suelen completar el programa propuesto (de las 23 bajas constatadas, una única era relacionada con el "grupo exclusivo”). Se ha indicado adicción a drogas y/o alcohol (37\%), psicopatología (9\%) y exposición a violencia familiar durante la infancia (15,5\%) en algunos casos.

La versatilidad de agresores supone una crítica a programas de tratamiento tan específicos o estandarizados (Véase Fernández-Montalvo \& Echeburúa, 2008; Loinaz, 2013). Consiguientemente, resultan interesantes los resultados del estudio realizado en el Centro Penitenciario Brians- 2 en Cataluña. Loinaz (2011) ha encontrado dos grupos de agresores: uno más homogéneo, que estaría compuesto por hombres violentos sólo con la pareja (“estables emocionalmente, integrados socialmente”), y otro más heterogéneo clasificado como violentos generalizados ("poco estables emocionalmente, no integrados socialmente”). El primer grupo (43\%), presenta mayor autoestima, personalidad compulsiva, narcisista e histriónica y generalmente tienen control de su ira. A pesar de llevar una vida considerada normal, estos sujetos explotan de forma desmesurada y habitualmente con su pareja. El segundo grupo (54\%), presenta mayor dependencia del alcohol y drogas, mayor incidencia de abusos en la infancia, mayor nivel de distorsiones cognitivas sobre la mujer y uso de violencia, mayor impulsividad, etc. Los agresores del segundo grupo, por tanto, presentan mayor número de factores de riesgo al ser comparados con el primer grupo (Véase también Gutiérrez, 2009).

En atención a estas tipologías, se sugiere el diseño de un programa de menor intensidad y de corta duración (con 15 sesiones, por ejemplo) para el grupo clasificado como violento solo con la pareja. Este programa debería estar centrado, entre otros aspectos, en la gestión y expresión de la ira, modificación de distorsiones cognitivas de la mujer, problemas asociados con la depresión, ansiedad y dependencia emocional. Ya para el grupo catalogado como agresores en general, los programas más adecuados serían aquellos con un número mayor de sesiones y direccionados a las distintas problemáticas antisociales de los individuos, como los tratamientos cognitivosconductuales centrados en cambiar la conducta violenta de los sujetos, vinculados al consumo de sustancia etc. Se ha comprobado, pues, que parte de los reclusos por violencia de género no serían personas con un estilo de vida considerado "normal” y 
cuya violencia surge en la intimidad del hogar o la relación de pareja. (Loinaz, 2011; Loinaz \& Echeburúa, 2010)

\subsection{Reincidencia}

En lo relativo a la reincidencia se ha encontrado un único estudio. Tras seguimiento de 40 reclusos que han cumplido pena en el Centro Penitenciario Brians- 2 por un tiempo medio de 15 meses, Loinaz (2011) ha descubierto que el 17,5\% ha reincidido ${ }^{4}$ tras la puesta en libertad y la mitad de este porcentaje lo ha hecho en un plazo inferior a los 7 meses. Los delitos cometidos tras la excarcelación han sido quebrantamiento, falta de lesiones, robo con fuerza, amenazas, violencia doméstica no habitual y lesiones. Por tanto, excluyendo el robo con violencia, se ha constatado que el 15\% ha reincidido en delitos contra la pareja. Ahora bien, es posible establecer una aproximación entre reincidencia y distintas tipologías de agresores y entre reincidencia y programas de rehabilitación. Sobre la relación reincidencia - tipologías, se ha observado que los agresores violentos, en general, han reincidido en mayor proporción que los violentos solo en la pareja (el 29\% de los reincidentes era de la tipología violento en la pareja).

En lo relativo a la relación reincidencia - programas de rehabilitación, se ha constatado que los sujetos tratados han reincidido significativamente menos que los no tratados (entre el grupo que había sido tratado apenas el 9,4\% ha cometido nuevo delito, mientras que el 50\% del grupo sin tratamiento ha reincidido). Como resultado global, los datos han revelado que no recibir tratamiento puede suponer una probabilidad del $50 \%$ de reincidir frente al 9\% de los que reciben tratamiento, así como que el 15\% de reincidencia específica en delitos de violencia contra la mujer puede ser considerado bajo si se compara con los estudios internacionales y con la cifra de reincidencia general de Cataluña (Loinaz, Irureta \& Doménech, 2011). En palabras de Loinaz (2011, p. 233), “el tratamiento parece tener un efecto potente en la reducción de la reincidencia, aunque aún debe establecerse qué factores influyen realmente en las nuevas agresiones, comparando características individuales con aspectos concretos del tratamiento” [cursiva añadida].

\footnotetext{
${ }^{4}$ El criterio utilizado para determinar la reincidencia es una nueva denuncia policial o nuevo ingreso en prisión habidos posteriormente a la finalización del programa.
} 


\subsection{Cambios cognitivos y psicopatológicos}

También se han destacado sobre los resultados de los programas las investigaciones a nivel cognitivo y psicopatológico tras la realización del tratamiento. En un estudio piloto, Echeburúa et al. (2006) han constatado que la intervención terapéutica ha proporcionado una mejora significativa de las creencias irracionales sobre las mujeres y la violencia como estrategia para hacer frente a las dificultades cotidianas, así como en lo relativo a la disminución de la sintomatología psicopatológica, ira y hostilidad. Al tiempo que los resultados del programa en su aspecto global han sido considerados positivos, no se sabe hasta qué punto pueden ser atribuidos al hecho de que el tratamiento rompa con la monotonía de la vida en prisión. La citada investigación conduce a resultados similares a la discusión de Echeburúa \& Fernández-Montalvo (2009) con una muestra más reveladora años más tarde. Sugieren que los agresores que han participado del tratamiento han experimentado una modificación significativa de las actitudes negativas sobre la mujer y sobre el uso de la violencia como forma válida de solución de conflictos. Igualmente, han constatado una mejora en el autocontrol de la expresión de la ira por los agresores, una reducción del grado de impulsividad y un aumento significativo de la autoestima. El programa de rehabilitación ha resultado efectivo en la medida que se han mejorado los síntomas psicopatológicos (somatización, depresión, ansiedad, ideación paranoide, entre otros) y, por tanto, los sujetos pueden presentar mayor control de los impulsos violentos.

Sin alejarse de los hallazgos anteriormente expuestos, Martínez et al. (2008) han descubierto que los resultados esperados del tratamiento en el Centro Penitenciario Quatre Camins en Cataluña han tenido apoyo empírico parcial. Ha sido posible atribuir al tratamiento los cambios en las distorsiones cognitivas, tanto hacia la mujer como hacia el uso de la violencia para resolver conflictos, así como la disminución de la impulsividad y de la ira. A pesar de que otras variables no hayan presentado diferencias importantes entre el grupo tratado y el grupo control, lo cierto es que el primero grupo han obtenido sistemáticamente mejores puntuaciones en relación a las variables psicológicas evaluadas (menores distorsiones cognitivas, mayor empatía, menor impulsividad, menor ira y mayor motivación terapéutica). A continuación, con una 
muestra de 28 agresores tratados, Martínez \& Pérez (2009) han puntualizado que las intervenciones psicológicas en prisión pueden ser eficaces en determinadas variables psicológicas, como la reducción de los impulsos. Esta nueva experiencia presenta un diferencial importante: los agresores participaron de programas de distintas intensidades. Concretamente, dos programas de baja intensidad (4 meses) para reclusos con condenas cortas y un programa más intenso (9 meses) destinado a los reclusos con condenas largas.

De una u otra manera, este conjunto de hallazgos positivos a nivel cognitivo y psicopatológico se han producido a su vez en programas intramuros con enfoque distinto del cognitivo-comportamental. Los agresores que han participado del PREMOVIGE, programa de enfoque emocional, han presentado mayor descenso de los pensamientos distorsionados sobre la mujer y sobre el uso de la violencia así como mayores expectativas de cambio (Rodríguez-Espartal \& López-Zafra, 2013). Ya aquellos que han participado de la Terapia de Aceptación y Compromiso (ACT) han presentado disminución de los niveles de evitación e impulsividad así como han bajado, ostensiblemente, el esfuerzo por eliminar el malestar, es decir, aceptación del malestar sin necesidad de reaccionar de una forma violenta (Flores \& Pascual, 2013) .

\section{Discusión y conclusiones}

\subsection{Discusión}

La presente revisión ha mostrado que desde las primeras experiencias rehabilitadoras puestas en marcha en España, el debate viene trasladándose de la necesidad de diseñar programas específicos de violencia de género que apuesten por el formato grupal y el enfoque cognitivo-conductual para adaptar las intervenciones a la pluralidad de agresores que forman parte del sistema penitenciario (Véase Echeburúa et al., 2006; Expósito \& Ruiz, 2010; Fernández-Montalvo \& Echeburúa, 2008; Fernández-Montalvo, Echauri, Martínez \& Azcárate, 2012; Loinaz et al., 2010; SGIP, 2011). La literatura reconoce los beneficios de esta metodología. Se destaca, por ejemplo, la posibilidad que tiene el terapeuta de gestionar de forma más segura los grupos, una vez que cuenta con participantes más similares y los esfuerzos pueden centrarse en aquellos sujetos que 
requieren más atención (Leganés, 2012). Desde esta perspectiva, además, se refuta la tesis de que la violencia de género es explicada solamente de acuerdo a valores culturales/machistas, aunque es cierto que en una sociedad cuya violencia se sustenta en pilares patriarcales los niveles de agresión contra la mujer tiendan a ser más elevados (Loinaz, 2009; 2013). En suma, los agresores son distintos y responden de manera diferente al mismo tratamiento (Cunha \& Gonçalves, 2013).

Ante el movimiento, a todas luces reciente, de adaptar las intervenciones intramuros a la pluralidad de agresores que han pasado a formar parte del sistema penitenciario, no sorprende que los programas hayan tomado por base una serie de datos y técnicas extraídas del ámbito comparado (Holtzworth-Munroe \& Meehan, 2004). Aunque las experiencias empíricas parecen comprobar la validez en el entorno español de los modelos internacionales de intervención, no se puede perder de vista que los sujetos pueden entrar con más facilidad en prisión (Llor-Esteban, García-Jiménez, RuizHernández \& Godoy-Fernández, 2015; Loinaz, 2013). Nadie ignora que tras la entrada en vigor de la LO 1/2004 las cárceles españolas han presenciado un vertiginoso aumento del ingreso de hombres por delitos y faltas en violencia de género. En cambio, pocos reconocen que se ha dado un empuje importante al diseño de programas intramuros. Desde una visión global es posible afirmar que el más influyente ha sido el Programa de Tratamiento en Prisión para Agresores en el Ámbito Familiar (SGIP, 2005) y que el surgimiento del actual Programa Marco PRIA (SGIP, 2010) no se debe al fracaso de los programas anteriores. Su objeto responde al intento de congregar las experiencias con el enfoque cognitivo-conductual desde la perspectiva de género. Se ha dado un paso en relación al plan ideológico pues parece ser que los programas anteriormente desarrollados se han caracterizado esencialmente por una orientación clínica (Expósito \& Ruiz, 2010). Aunque los resultados del Programa Marco PRIA en medio cerrado no han sido publicados, imposibilitando cualquier discusión sobre el impacto del nuevo enfoque propuesto, las investigaciones recogidas en el presente estudio sugieren que, más que el enfoque adoptado, lo diferencial es que el sujeto haya recibido un programa y preferentemente próximo a su excarcelación (Flores \& Pascual, 2013; Rodríguez-Espartal \& Lopez-Zafra, 2013).

En la práctica existe un porcentaje substancial de agresores que entran en prisión para cumplir penas relativamente cortas (entre los 6 meses y 2 años) y que 
acaban sin poder participar en una intervención (Sordi, 2014). Un cuerpo robusto de investigaciones ha acreditado que la cárcel no contribuye, por sí misma, a reducir la ocurrencia de actos de violencia de género (Pascual-Leone, Bierman, Arnold \& Stasiake, 2011; Ventura \& Davis, 2005). Esta puede proveer un efecto disuasorio momentáneo, pero difícilmente evitará que se produzcan nuevas agresiones a las mujeres pareja, sean estas actuales, pasadas o futuras, si no se trabaja con el agresor (Day, Richardson, Bowen \& Bernardi, 2014). A propósito, en algunos casos, el paso por la prisión incrementa el riesgo de la ocurrencia de agresiones más graves. Es necesario, pues, implementar tanto estrategias para reforzar una política criminal que trate de reducir el uso de la prisión en favor de respuestas que no supongan la privación de cuotas de libertad (Cid, 2007; 2009) como programas de corta duración en medio cerrado (Martínez \& Pérez, 2009). La reducción de la intervención puede parecer una falta de rigor profesional, pero lo cierto es que no existen pruebas sobre el incremento de la efectividad con base apenas en la extensión temporal del programa (Expósito \& Ruiz, 2010). Lo que sí parece influyente es que el programa sea conducido por profesionales de ambos sexos (Expósito \& Ruiz, 2010), suficientemente cualificados para incrementar la motivación de los internos en las primeras sesiones a fin de minimizar los índices de abandono (Echeburúa et al., 2006; Filardo, 2013).

Consiguientemente, al tiempo que se deben fomentar las alternativas a la vía penal y la intervención temprana sobre la violencia, no se pueden obviar o minimizar las variables psicológicas del sujeto que agrede (Véase Fernández -Montalvo \& Echeburúa, 2008; García-Jiménez, Sánchez-Meca \& Godoy-Fernández, 2013; Loinaz \& Echeburúa, 2010; 2012). Prueba de lo dicho es que el conjunto de investigaciones aquí analizadas demuestran que algunos maltratadores sí son capaces de una transformación personal (Expósito \& Ruiz, 2010). Se ha comprobado que un programa en medio cerrado posibilita mejoras en las distorsiones cognitivas hacia la mujer y hacia el uso de la violencia para resolver conflictos, así como en la disminución de la impulsividad y en el control de la ira (Echeburúa et al., 2006; Echeburúa \& Fernández-Montalvo, 2009; Martínez et al., 2008; Martínez \& Pérez, 2009; Rodríguez-Espartal \& Lopez-Zafra, 2013). En este contexto, los estudios epidemiológicos sobre agresores se vienen mostrando un aliado potente de los programas específicos en violencia de género al sugerir, entre otras cuestiones, la necesidad de combinarlos con otros programas, como 
los dedicados al consumo de alcohol y drogas. En una muestra de 106 reclusos por violencia de género, Chérrez-Bermejo \& Alás-Brun (2014) han dado a conocer que el porcentaje de abuso de sustancias llega al 61,3\%, siendo el alcohol y la cocaína las substancias de mayor uso. Este porcentaje es mayor que en otros estudios realizados sobre poblaciones en situación de prisión. Además, han precisado que, al ingreso en prisión, no pocos agresores tienen, al menos, un diagnóstico psiquiátrico (25,5\%), escasamente conocido por los servicios de salud mental comunitarios. En otras palabras, la salud mental de los agresores parece ser un problema penitenciario.

Los programas españoles deben adoptar una postura más realista para que se inviertan esfuerzos en logros alcanzables (Martínez \& Pérez, 2009). Dicho en otros términos, definir los indicadores que evalúan el programa es tan importante como definir el diseño del programa en sí mismo, incluso con datos que, como la reincidencia, parecen a primera vista objetivos. La reincidencia es considerada el índice por excelencia de los resultados de un programa, pero se puede definir de forma dispar: con base en la palabra de la víctima, por medio de un nuevo registro policial o por el ingreso en prisión (Saunders, 2008). Por tanto, el problema más evidente alrededor de la medición de los resultados de los programas para agresores es establecer qué evaluar, cómo evaluar y cuándo evaluar (Gondolf, 2002; 2012). Cuestiones como el tamaño de la muestra, el contar con grupo control y el tiempo de seguimiento vienen mostrándose determinantes para la validación de los resultados (Babcock, Green \& Robie, 2004; Gondolf, 2002; 2004). En España, todavía, estos parámetros necesitan ser consideradas con mayor rigor. Tomando en cuenta esta realidad, se puede afirmar que los datos empíricos disponibles sobre los resultados de los programas intramuros no ofrecen resultados concluyentes (Echeburúa \& Fernández-Montalvo, 2009).

Es particularmente interesante que al confrontar los datos españoles disponibles con las investigaciones internacionales sobre el tema, se constata una cierta similitud entre los resultados encontrados. ${ }^{5}$ Tras una evaluación pre y postratamiento,

\footnotetext{
${ }^{5}$ Las investigaciones más influyentes sobre programas para agresores de violencia de género en ámbito cerrado provienen de Canadá. Los Servicios Correccionales (Correctional Service of Canada) han implementado un proceso de acreditación de programas con el propósito de asegurar que los mismos sean adecuados y eficaces para reducir las tasas de reincidencia (Standards for Correctional Programs). En el año 2001 se acreditó el Programa de Prevención de la Violencia Familiar (Family Violence Prevention Programs), que puede desarrollarse en distintas intensidades en función del riesgo existente de que el agresor cometa nuevos actos violentos y del lugar dónde tienen espacio, institución cerrada o medio
} 
Stewart, Gabora, Kropp \& Lee (2005) han demostrado que la participación en un programa de moderada o alta intensidad reduce significativamente las actitudes que apoyan la violencia contra las mujeres y aumenta las habilidades pro-sociales de los sujetos. Estos hallazgos se han confirmado tanto en los testimonios de los agentes de libertad condicional responsables de supervisar a los agresores una vez finalizado el tratamiento como durante el período de seguimiento (6 meses contados de la puesta en libertad). Entre sus conclusiones se destacan dos puntos. Primeramente, que aquellos que han completado uno de los programas han tenido 4 veces menos probabilidades de cometer un acto de violencia conyugal, contribuyendo la finalización del tratamiento a la reducción de otros tipos de violencia. De otra parte, no ha sido posible comprobar que la mayor duración del tratamiento e intensidad del programa hayan implicado necesariamente una mejora en los resultados (Véase también Kropp \& Lee, 2004). La investigación de Connors, Mills \& Gray (2012), con 298 sujetos que han participado de un programa de moderada intensidad, viene a corroborar los datos obtenidos años antes. Los reclusos han presentado mejoras tras el tratamiento, tanto en lo relativo a la adquisición de habilidades y cambios de actitudes (reducción de los celos, ira, dependencia) como en la mayor aceptación de la responsabilidad por los hechos violentos. Resaltan los investigadores, sin embargo, que no se han producido todos los cambios esperados por el programa. Además, han hecho hincapié en la motivación de los penados para participar del mismo: si bien a veces se ha mostrado problemática, lo cierto es que durante la intervención se ha incrementado.

En conclusión, aún sin poder extraer resultados irrefutables, el conjunto de investigaciones analizadas en el presente estudio coinciden en que los programas en prisión para autores de violencia contra la pareja o ex pareja son prometedores para la reducción de las actitudes abusivas y la violencia entre íntimos. En España, sin embargo, los resultados alcanzables por las intervenciones intramuros de acuerdo a las necesidades de la población reclusa parecen haber sido relegados a un segundo plano

comunitario. La intervención de alta intensidad está diseñada en 9 módulos, en grupos de 12 participantes, tiene un mínimo de 164 horas de duración y está diseñado para el ámbito cerrado. Ya el programa de moderada intensidad está diseñado en 6 módulos para que pueda ser ejecutado en un tiempo total de 75 horas de duración y puede tener lugar en el ámbito cerrado o comunitario (Correctional Service Canada, 2001). Según declaran los Servicios Correccionales, citado Programa Marco tiene aplicación no solo en territorio canadiense, sino también en otros países de habla inglesa, concretamente Estados Unidos e Inglaterra (Correctional Service Canada, 2009). 
por parte de las autoridades penitenciarias, pues la evolución de estos programas no ha venido acompañada de un estudio riguroso que contemple, entre otra cuestiones, muestra de gran amplitud, grupo control y seguimiento. En efecto, se ha tenido escasamente en cuenta que la evidencia científica es un importante motor de avance para la sofisticación de los programas y para evitar los riesgos de nuevas victimizaciones. Igualmente, que contribuye al ahorro de los recursos públicos si las inversiones se destinan a programas que hayan demostrado resultados positivos y/o se orientan a los contenidos del programa que se han mostrado más importantes para el cambio y prevención de reincidencia. Desde una perspectiva optimista, quisiéramos resaltar que la existencia de conocimiento científico aún por producirse no significa que no hayan existido importantes avances. De hecho, el presente trabajo ha revelado que la comunidad científica viene realizando notables esfuerzos para identificar la intervención más adecuada. Entre otras cuestiones, se ha comprobado que algunos de los agresores tienen perfil psicopatológico y sesgos cognitivos específicos $\mathrm{y}$, por tanto, los tratamientos psicológicos intramuros pueden proporcionar buenos resultados para determinados tipos de agresores. Como efecto reflejo, se revela que la existencia de una estructura jurídica - art. 42 LO 1/2004 - que determina que se realicen programas dentro de las prisiones y que exige respetar la voluntariedad de los penados en participar del tratamiento - art. 112 Reglamento Penitenciario - no implica que se deba enviar a todo agresor a participar de un mismo programa, poniendo a prueba los programas estándares.

En definitiva, los hallazgos aquí recogidos demuestran que hay que seguir apostando por los programas intramuros. El compromiso es arrojar luz sobre las evidencias resultantes del tratamiento con el mayor grado de fiabilidad posible.

\subsection{Limitaciones e investigación futura}

Este estudio pretende ser una primera aproximación al análisis conjunto de la literatura científica sobre hombres en situación de prisión en España por delitos relacionados con violencia de género que cumplieron o estaban cumpliendo un programa de rehabilitación específico intramuros. En este sentido, es importante señalar que a pesar del intento significativo de recoger el mayor número de publicaciones sobre el tema, no 
se ha incluido la llamada literatura gris (como actas de jornadas y encuentros, editoriales, entre otros). Además, la literatura usada en el presente estudio se ha valido de distintas metodologías y no necesariamente contempla variables coincidentes, circunstancias que limitan tanto el análisis conjunto de los datos como conclusiones con mayor rigor científico. La dificultad de analizar conjuntamente las investigaciones seleccionadas es un hecho consecuencia de las distintas metodologías utilizadas. Asimismo, las investigaciones que contemplan variables diferenciales tipológicas y seguimiento a largo plazo son exiguas. Tampoco se ha publicado un análisis trasversal de los programas bajo la competencia de la SGIP y los realizados en Cataluña, aunque se hayan adoptado puntos de partida diversos, dado que esta Comunidad Autónoma ha implantado en medio cerrado programas con distintas intensidades adaptados a las tipologías de los agresores. Hay que aclarar, igualmente, que no todos reclusos estaban participando o habían participado del mismo programa.

Consiguientemente, sería interesante que se realizara una investigación futura sobre el eventual impacto del diseño de los programas en los resultados postratamiento y otra, en profundidad, sobre las variables e instrumentos utilizados en las evaluaciones pre y postratamiento. Al propio tiempo, sería relevante un estudio metaanalítico que permitiera el análisis conjunto de las evidencias disponibles sobre los programas intramuros. También es importante señalar que las búsquedas para este estudio se han llevado a cabo durante el año de 2013 y que una actualización de las mismas ha sido realizada en julio de 2015. Por tanto, podrían surgir posibles limitaciones bien de la existencia de investigaciones a la espera de un parecer positivo para publicación, bien del retraso existente en la incorporación de los estudios en las bases de datos. En cualquier caso, puede afirmarse que se ha realizado una gran búsqueda con el fin, al menos, de lograr una primera aproximación al tema. Se espera que las revisiones futuras puedan contar con estudios más coincidentes, capaces de arrojar luz sobre categorías científicas atinentes al tema en discusión.

\section{Agradecimientos}

Quiero agradecer a Borja Mapelli Caffarena (Universidad de Sevilla), Juan José Medina Ariza (University of Manchester) y Concepción Yagüe (Secretaría General de 
Instituciones Penitenciarias) las interminables discusiones anteriores a la realización de este texto. A los evaluadores y editor-jefe de REIC mis agradecimientos por las sugerencias y observaciones que me han permitido mejorar el artículo.

\section{Financiación}

El presente trabajo ha sido posible gracias a la Agencia Española para la Cooperación Internacional y el Desarrollo - Ministerio de Asuntos Exteriores y de Cooperación (becas MAEC-AECID).

\section{Referencias}

Babcock, J., Green, C. \& Robie, C. (2004). Does batterers' treatment work? A metaanalytic review of domestic violence treatment. Clinical Psychology Review, 23(8), 1023-1053.

Chérrez-Bermejo, C. \& Alás-Brun, R. (2014). Consumo de sustancias y trastornos de salud mental en agresores de violencia de género ingresados en prisión. Un estudio descriptivo. Revista Española de Sanidad Penitenciaria, 16, 29-37.

Cid, J. (2007). ¿Es la prisión criminógena? (un análisis comparativo de reincidencia entre la pena de prisión y la suspensión de la pena). Revista de Derecho Penal y Criminología, 19, 427-456.

Cid, J. (2009). La elección del castigo. Barcelona: Bosch.

Connors, A., Mills, J. \& Gray, A. (2012). An Evaluation of Intimate Partner Violence Intervention with Incarcerated Offenders. Journal of Interpersonal Violence, 27(6), 1176-1196.

Correctional Service Canada (2001. Revised 2006). Assessment manual for the moderate and high intensity family violence prevention programs. Reintegration Programs Division. Disponible en http://www.cscscc.gc.ca/text/prgrm/cor-pro-2009-eng.shtml\#_ftnref17

Correctional Service Canada (2009). Correctional Program Descriptions. Reintegration Programs Division. Disponible en http://www.csc-scc.gc.ca/text/prgrm/corpro-2009-eng.shtml\#_ftnref17.

Cunha, O. \& Gonçalves, R. A. (2013). Intimate partner violence offenders: Generating a data-based typology of batterers and implications for treatment. The European Journal of Psychology Applied to Legal Context, 5(2), 131-139. 
Day, A., Richardson, T., Bowen, E. \& Bernardi, J. (2014). Intimate partner violence in prisoners: Toward effective assessment and intervention. Aggression and Violent Behavior, 19, 579-583.

Echauri, J. A., Fernández-Montalvo, J., Martínez, M. A. \& Azcárate, J. M. (2011). Trastornos de Personalidad en Hombres Maltratadores a la Pareja: Perfil Diferencial entre Agresores en Prisión y Agresores con Suspensión de Condena. Anuario de Psicología Jurídica, 21, 97-105.

Echauri, J. A., Romero, J. \& Rodríguez, M. J. (2005). Teoría y descripción de la violencia doméstica: programa terapéutico para maltratadores del ámbito familiar en el centro penitenciario de Pamplona. Anuario de Psicología Jurídica, 15, 67-95.

Echeburúa, E. \& Fernández -Montalvo, J. (2009). Evaluación de un programa de tratamiento en prisión de hombres condenados por violencia grave contra la pareja. International Journal of Clinical and Health Psychology, 9(1), 5-20.

Echeburúa, E., Fernández -Montalvo, J. \& Amor, P. (2006). Psychological treatment of men convicted of gender violence: A pilot study in Spanish prisons. International Journal of Offender Therapy and Comparative Criminology, 50, 57-70.

Expósito, F. \& Ruiz, S. (2010). Reeducación de Maltratadores: Una Experiencia de Intervención desde la Perspectiva de Género. Intervención Psicosocial, 19(2), 45-151.

Fernández-Montalvo, J. (2009). La violencia de género y los trastornos de personalidad. Jano. Medicina y Humanidades, 1752, 21-24.

Fernández -Montalvo, J., Echauri, J., Martínez, M. \& Azcárate, J. M. (2012). Batterer Men in Prison and in Court-Referred Treatment Programmes: What is the Difference?. The Spanish Journal of Psychology, 15(1), 315-322.

Fernández-Montalvo, J. \& Echeburúa, E. (2005). Hombres condenados por violencia grave contra la pareja: un estudio psicopatológico. Análisis $y$ Modificaciones de Conducta, 31(138), 451- 475.

Fernández-Montalvo, J. \& Echeburúa, E. (2008). Trastornos de personalidad y psicopatía en hombres condenados por violencia grave contra la pareja. Psicothema, 20(2), 193-198.

Filardo, C. (2013). Intervención desde el trabajo social con hombres maltratadores en los centros penitenciarios españoles. Documentos de Trabajo Social, 52, 930 .

Flores, L. M. S. \& Pascual, C. F. S. (2013). Aplicación de la Terapia de Aceptación y

Revista Española de Investigación Criminológica

Artículo 6, Número 13 (2015)

www.criminologia.net

ISSN: 1696-9219 
Compromiso (ACT) con hombres que cumplen condena por maltrato. Un estudio piloto. International Journal of Psychology and Psychological Therapy, 13(3), 289-305.

García, E. \& Díez-Ripollés, J. L. (Dir.) (2012). Realidad y política penitenciarias. Informe ODA 2010/2011. Málaga: Instituto Andaluz Interuniversitario de Criminología.

García-Jiménez, J. J., Sánchez-Meca, J. \& Godoy-Fernández, C. (2013). Distorsiones cognitivas respecto a la violencia de género en presos. En F. Expósito, I. Valor-Segura, M. Vilariño \& A. Palmer (Eds.), Psicología jurídica aplicada a los problemas sociales (pp. 89-96). Madrid: Sociedad Española de Psicología Jurídica y Forense.

Gondolf, E. (2002). Batterer Intervention Systems. Issues, Outcomes and Recommendations. London: Sage.

Gondolf, E. (2004). Evaluating batterer counseling programs: A difficult task showing some effects and implications. Aggression and Violent Behavior, 9, 605631.

Gondolf, E. (2012). The future of batterer programs: reassessing evidence-based practice. Northeastern University Press.

Gutiérrez, M. C. (2009). Programa de Tratamiento en Prisión para Agresores en el Ámbito Familiar. Comunicación presentada en el VI Congreso Español de Criminología, Criminalidad y Riesgo: Predicción y Prevención, 19 - 20 de Junio. Santiago de Compostela: Universidad de Santiago de Compostela, p. 48.

Disponible en: http://www.criminologia.net/pdf/congresosSEIC/libro_actas_Congreso2009. pdf

Holtzworth-Munroe, A. \& Meehan, J. C. (2004). Typologies of Men Who Are Maritally Violent: Scientific and Clinical Implications. Journal of Interpersonal Violence, 19(12), 1369-1389.

Instituto Andaluz de la Mujer (2012). La Renuncia a continuar en el Procedimiento Judicial en Mujeres Víctimas de Violencia de Género: Un estudio en la Comunidad Autónoma Andaluza. Sevilla: Instituto Andaluz de la Mujer.

Kropp, P. R. \& Lee, Z. (2004). Evaluation of the Correctional Service of Canada Family Violence Prevention Programs: Final report. Institute Against Family Violence. Disponible en http://www.cscscc.gc.ca/publications/index-eng.shtml

Leganés, S. (2012). Evolución de los programas para agresores. En E. Martínez García \& J. C. Vegas Aguilar, La ejecución de los trabajos en beneficio de la 
comunidad por delitos de violencia de género (pp. 102- 107). Valencia: Tirant lo Blanch.

Llor-Esteban, B., García-Jiménez, J. J., Ruiz-Hernández, J. A. \& Godoy-Fernández, C. (2015). Profile of partner aggressors as a function of risk of recidivism. International Journal of Clinical and Health Psychology, in press.

Loinaz, I. (2009). Aproximación teórica y empírica al estudio de las tipologías de agresores de pareja: análisis descriptivo de variables e instrumentos de evaluación en el centro penitenciario Brians-2. Madrid: Ministerio del Interior.

Loinaz, I. (2011). Clasificación de agresores de pareja en prisión. Implicaciones terapéuticas y de gestión de riesgo. En Intervención con agresores de violencia de género (pp. 153-276). Barcelona: Centre d' Estudis Jurídics i Formació Especialitzada, Generalitat de Cataluña.

Loinaz, I. (2013). Carrera delictiva y reincidencia en agresores de pareja en prisión. En R. Castillejo Manzanares \& C. Alonso Salgado, Violencia de género y Justicia (pp. 647-676). Santiago de Compostela: Universidad de Santiago de Compostela.

Loinaz, I. \& Echeburúa, E. (2010). Necesidades terapéuticas en agresores de pareja según su perfil diferencial. Clínica Contemporánea, 1(2), 85-95.

Loinaz, I. \& Echeburúa, E. (2012). Apego adulto en agresores de pareja. Acción Psicológica, 9(1), 33-46.

Loinaz, I., Echeburúa, E. \& Torrubia, R. (2010). Tipología de agresores contra la pareja en prisión. Psicothema, 22(1), 106-111.

Loinaz, I., Irureta, M. \& Doménech F. (2011). Análisis de la reincidencia en agresores de pareja. Documentos de Trabajo, Investigación: Àmbit social i criminològic. Barcelona: Centre d’Estudis Jurídics i Formació Especialitzada.

Martínez, M. \& Pérez, M. (2009). Evaluación criminológica y psicológica de los agresores domésticos. Documentos de Trabajo, Investigación: Àmbit social i criminològic. Barcelona: Centre d'Estudis Jurídics i Formació Especialitzada.

Martínez, M., Pérez, M. \& López, J. M. (2008). Avaluació del programa de tractament d'agressors domèstics. Documents de Treball, Investigació: Àmbit social i criminològic. Barcelona: Centre d’Estudis Jurídics i Formació Especialitzada.. 
Pascual-Leone, A., Bierman, R., Arnold, R. \& Stasiak, E. (2011). Emotion-focused therapy for incarcerated offenders of intimate partner violence: A 3-year outcome using a new whole-sample matching method. Psychotherapy Research, 21(3), 331-347.

Rodríguez-Espartal, N. \& Lopez-Zafra, E. (2013). Programa emocional para presos por violencia de género (PREMOVIGE): Efectividad en variables cognitivas y conductuales. Psychosocial Intervention, 22(2), 115-124.

Saunders, D. (2008). Group Interventions for Men Who Batter: A Summary of Program Descriptions and Research. Violence and Victims, 23(2), 156-172.

Secretaría General de Instituciones Penitenciarias. (SGIP). (2005). Documentos Penitenciarios 2. Programa de Tratamiento en Prisión para agresores en el ámbito familiar. Grupo de trabajo sobre violencia de género. Madrid: Ministerio del Interior. Disponible en http://www.institucionpenitenciaria.es/

Secretaría General de Instituciones Penitenciarias. (SGIP). (2010). Documentos Penitenciarios 7. Violencia de Género. Programa de Intervención para Agresores - PRIA. Madrid: Ministerio del Interior. Disponible en http://www.institucionpenitenciaria.es/

Secretaría General de Instituciones Penitenciarias. (SGIP). (2010a). El delincuente de género en prisión. Estudio de las características personales $y$ criminológicas y la intervención en el medio penitenciario. Madrid: Ministerio del Interior. Disponible en http://www.institucionpenitenciaria.es

Secretaría General de Instituciones Penitenciarias. (SGIP). (2011). El Delito de Violencia de Género y los Penados Extranjeros anexo al Documentos Penitenciarios 7. Violencia de Género. Programa de Intervención para Agresores. Madrid: Ministerio del Interior. Disponible en http://www.institucionpenitenciaria.es

Secretaría General de Instituciones Penitenciarias. (SGIP). (2012). Informe general de 2011. Madrid: Ministerio del Interior. Disponible en http://www.institucionpenitenciaria.es/web/portal/documentos/publicacione s.html

Secretaría General de Instituciones Penitenciarias. (SGIP). (2015). Tipología Delictiva de la Población Reclusa Penada Ley Orgánica 10/1995, de 23 de Noviembre, del Código Penal, Mensual, julio de 2015, Total Nacional. Madrid: Ministerio del Interior. Disponible en http://www.institucionpenitenciaria.es/web/portal/documentos/estadisticas.h $\mathrm{tml}$ ? $\mathrm{r}=\mathrm{m} \& \mathrm{adm}=\mathrm{TES} \& \mathrm{am}=2015 \& \mathrm{~mm}=7 \& \mathrm{tm}=\mathrm{TIP} 1 \& \mathrm{tm} 2=\mathrm{GENE}$ 
Sordi, B. (2014). Análisis político criminal de los programas de rehabilitación para agresores de violencia de género. [Tesis doctoral inédita]. Universidad de Sevilla, Facultad de Derecho, España.

Stewart, L., Gabora, N., Kropp, R. \& Lee, Z. (2005. Revised 2008). Family Violence Programming: Treatment Outcome for Canadian Federally Sentenced Offenders. Institute Against Family Violence. Correctional Operations and Programs, Reintegration Programs Division, Correction Service Canada. Research Report N R-174, 2005. Disponible en http://www.cscscc.gc.ca/research/r174-eng.shtml

Ventura, L. A. \& Davis, G. (2005). Domestic Violence Court Case Conviction and Recidivism. Violence Against Women, 11(2), 255-277.

Bárbara Sordi Stock es doctora por la Universidad de Sevilla (Doctorado con Mención Internacional, University of Manchester) y ha trabajado como docente e investigadora en UniRitter- Brasil y el Instituto Andaluz Interuniversitario de Criminología Universidad de Sevilla. En la actualidad, es Experta COMJIB - EUROsociAL II Programa Apoyo al Acceso a la Justicia. Violencia de género en Iberoamérica: investigación de delitos, atención a víctimas y coordinación interinstitucional. Su principal área de interés es género y sistema de justicia penal. 